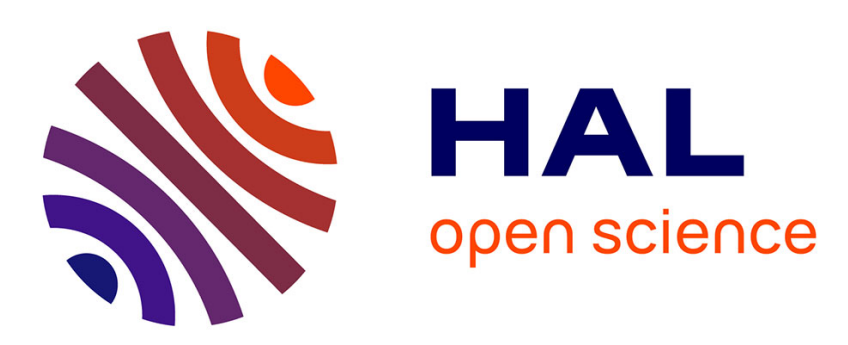

\title{
Application d'une méthode de perturbation à l'étude de résonateurs à quartz présentant des modes d'épaisseur à énergie piégée
}

B. Dulmet

\section{- To cite this version:}

B. Dulmet. Application d'une méthode de perturbation à l'étude de résonateurs à quartz présentant des modes d'épaisseur à énergie piégée. Revue de Physique Appliquée, 1984, 19 (9), pp.839-849. 10.1051/rphysap:01984001909083900 . jpa-00245274

\section{HAL Id: jpa-00245274 \\ https://hal.science/jpa-00245274}

Submitted on 1 Jan 1984

HAL is a multi-disciplinary open access archive for the deposit and dissemination of scientific research documents, whether they are published or not. The documents may come from teaching and research institutions in France or abroad, or from public or private research centers.
L'archive ouverte pluridisciplinaire HAL, est destinée au dépôt et à la diffusion de documents scientifiques de niveau recherche, publiés ou non, émanant des établissements d'enseignement et de recherche français ou étrangers, des laboratoires publics ou privés. 


\title{
Application d'une méthode de perturbation à l'étude de résonateurs à quartz présentant des modes d'épaisseur à énergie piégée
}

\author{
B. Dulmet
}

Laboratoire de Chronométrie, Electronique et Piézoélectricité de l'Ecole Nationale Supérieure de Mécanique et des Microtechniques, La Bouloie, Route de Gray, 25030 Besançon Cedex, France

(Reçu le 15 mars 1984, révisé le 2 mai, accepté le 15 juin 1984)

\begin{abstract}
Résumé. - L'élaboration d'un modèle analytique simple pour la description des vibrations d'un résonateur à énergie piégée nécessite l'abandon de nombreux coefficients élastiques. Nous proposons une méthode de perturbation pour la prise en considération des principaux d'entre eux. Nous mettons ainsi en évidence les mécanismes de couplages entre modes, dont l'effet sur la fréquence est évalué, pour des caractéristiques données du résonateur.

Abstract. - Many elastic coefficients must be ignored in the elaboration of a suitable model for contoured quartz resonators vibrating in thickness modes. A perturbation analysis is proposed, which takes into account the most significant coefficients. Taking into account the geometry of well defined resonators we clarify the mechanism of couplings between the modes, whose influence on the frequency is obtained.
\end{abstract}

\section{Introduction.}

Dans la gamme des fréquences comprises entre 1 et $10 \mathrm{MHz}$, les modes résonnants les plus employés dans les résonateurs métrologiques à ondes de volume, sont les modes dits à énergie piégée. Dans le cas de résonateurs plans-convexes ou biconvexes, ces modes correspondent à des ondes acoustiques se propageant essentiellement suivant la direction de la normale à la plaque de quartz et subissant une dispersion provoquée par la variation lente de l'épaisseur du cristal. Il existe divers modèles pour la description de ce type de vibration [1-3]. Les modes sont caractérisés par une composante en épaisseur du vecteur d'onde, qui tend asymptotiquement vers la valeur prise dans le cas d'une plaque infinie de même fréquence [4]. Une hypothèse simplificatrice consiste à attribuer à la polarisation de l'onde acoustique du mode piégé les composantes qu'elle présenterait dans le cas d'une véritable onde plane $[2,5]$. Un tel modèle, introduit pour la première fois par $\mathrm{C}$. J. Wilson pour l'étude des coupes $Y+\theta$ [2], nous donnera les modes « non perturbés ». Ils se présentent sous forme de familles découplées (compression $A$, cisaillement rapide $B$, cisaillement lent $C$ ) obéissant à des répartitions harmoniques suivant l'épaisseur, et en fonctions d'Hermite dans le plan de la plaque. Généralisable à toutes les orientations cristallines [5], ce modèle nécessite l'abandon de nombreux coefficients élas- tiques, dont les valeurs numériques varient en fonction de l'orientation de la plaque, ce qui se répercute sur la précision. Nous utilisons une méthode de perturbation $[6,7]$ pour tenir compte des coefficients supplémentaires, en négligeant seulement des coefficients dont l'effet reste secondaire par suite de la géométrie du résonateur (plaque mince). Les coefficients supplémentaires traduisent l'existence de couplages entre modes piégés, d'une même famille ou non. On doit poursuivre les développements au second ordre. En conséquence, la perturbation sur la fréquence ne peut être obtenue que numériquement.

L'ampleur des perturbations calculées nous éclaire sur l'aptitude du modèle de Wilson, à rendre compte d'une configuration donnée.

\section{Equations de base.}

Pour obtenir les équations donnant accès aux modes simples non perturbés, nous suivons d'assez près la démarche adoptée par H. F. Tiersten et D. S. Stevens dans plusieurs articles récents $[3,8]$.

D'abord, on examine le cas d'une plaque dont les dimensions latérales sont infinies au regard de l'épaisseur. On adopte un premier repère de travail, $\hat{R}$, lié à la plaque conformément aux dispositions de la figure 1. Nous négligeons l'influence de la piézoélectricité. Dans le repère $\hat{R}$, les équations de l'élasti- 


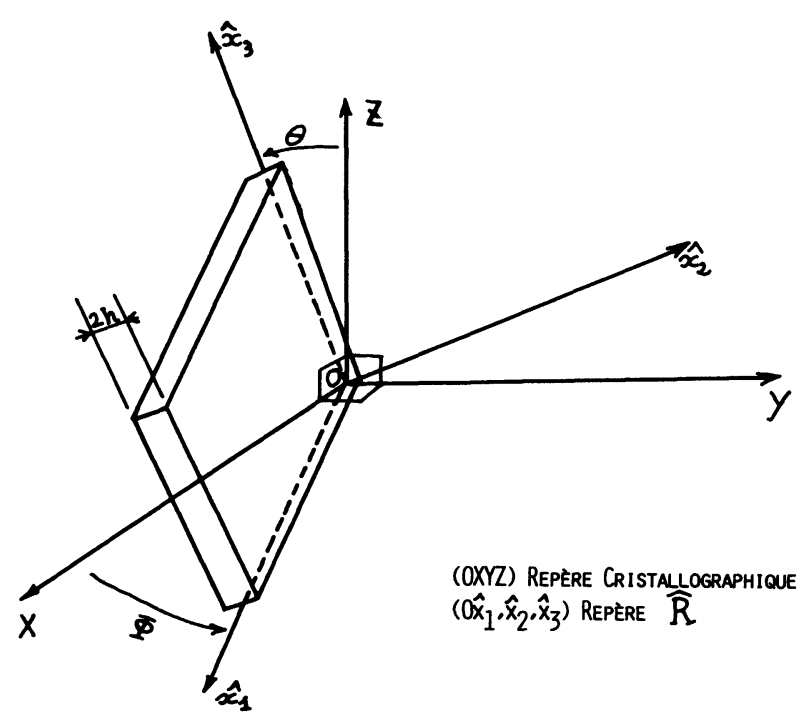

Fig. 1. - Plaque en coupe à double rotation.

[Doubly rotated cut.]

cité, dans le cadre de l'approximation linéaire, se réduisent à :

$$
\left\{\begin{array}{l}
\hat{T}_{j i}=\hat{C}_{j i k l} \frac{\delta \hat{u}_{l}}{\delta \hat{x}_{k}} \\
\frac{\partial \hat{T}_{j i}}{\partial x_{j}}=\rho \ddot{\hat{u}}_{i}
\end{array} i, j, k, l=1,2,3 .\right.
$$

$\hat{u}_{i}$ est une composante du déplacement particulaire dans le repère $\hat{R}$, lié aux axes de symétrie du cristal par les angles de coupe $\theta$ et $\Phi ; \hat{T}_{j i}$ est un élément du tenseur des efforts et $\hat{x}_{j}$ une coordonnée courante dans $\hat{R}$. $\rho$ est la masse volumique du matériau. Les valeurs des coefficients élastiques $\hat{C}_{i j k l}$ peuvent être obtenues à partir des valeurs tabulées dans le repère cristallographique, par simple rotation tensorielle. Nous travaillons d'abord avec des indices non réduits. variant donc de un à trois, et nous adoptons la convention de sommation des indices muets répétés.

Une plaque infinie peut être le siège de trois ondes planes se propageant normalement à son plan [9]. Chacune de ces trois ondes, que nous distinguerons par la valeur 1,2 ou 3 , arbitrairement attribuée au symbole $\alpha$, correspond au déplacement :

$$
\hat{u}_{k}^{\alpha}=A_{k}^{\alpha} \exp \left(j \omega\left(t-\hat{x}_{2} / V^{\alpha}\right)\right) \quad(k=1,2,3) .
$$

La normale à la plaque correspondant à la direction $\hat{x}_{2}$, le caractère infini des dimensions latérales entraîne la nullité des variations des grandeurs mécaniques ou électriques dans le plan $\left(\hat{x}_{1} \mathrm{O} \hat{x}_{3}\right)$.

$V^{\alpha}$ est la vitesse de phase du mode $\alpha, A_{k}^{\alpha}$ est une des composantes de son amplitude dans le repère $\hat{R}$. En résolvant le système linéaire suivant :

$$
\left[\hat{C}_{2 i 2 l}-\hat{C}^{\alpha} \delta i l\right]\left[A_{l}^{\alpha}\right]=[0]
$$

dont l'équation aux valeurs propres est :

$$
\left|\hat{C}_{2 i 2 l}-C^{\alpha} \delta i l\right|=0
$$

on obtient les valeurs propres $C^{\alpha}$, dites coefficients de la propagation, qui donnent accès aux vitesses $V^{\alpha}$, sachant que $C^{\alpha}=\rho\left(V^{\alpha}\right)^{2}$. Les vecteurs propres $\mathbf{A}^{\alpha}$ associés aux coefficients $C^{\alpha}$ peuvent être normés et rangés de manière à former un trièdre orthonormé $\left(\mathbf{A}^{1}, \mathbf{A}^{2}, \mathbf{A}^{3}\right)$. Les trois vecteurs $\mathbf{A}^{\alpha}$ constituent une matrice orthogonale $Q$ dont lëlément générique est :

$$
Q_{x j}=A_{j}^{x} ; \quad[Q]^{T}=[Q]^{-1} .
$$

La matrice $Q$ ne dépend que de l'orientation de la plaque ainsi que des coefficients élastiques et de la masse volumique du matériau. Un vecteur $\mathbf{V}$ quelconque de composantes $\hat{v}_{i}$ dans le repère $\hat{R}$, peut également être représenté par ses composantes $v_{i}$ sur la base des $\mathbf{A}^{\alpha}$. Les lois de transformations sont les suivantes :

$$
v_{i}=Q_{i r} \hat{v}_{r} ; \hat{v}_{r}=Q_{i r} v_{i} .
$$

Par ailleurs, nous pouvons observer que, par rotation arbitraire d'angle $\gamma$ autour de $\hat{x}_{2}$, le repère $\hat{R}$ se transforme en un nouveau repère $\mathcal{R}$, dans lequel les coordonnées courantes $x_{i}$ sont liées au $\hat{x}_{j}$ par :

$$
x_{i}=R_{i j} \hat{x}_{j} ; \quad \hat{x}_{j}=R_{i j} x_{i} ; \quad \frac{\partial}{\partial \hat{x}_{j}}=R_{i j} \frac{\partial}{\partial x_{i}} .
$$

La matrice de rotation $R$ est la suivante :

$$
[R]=\left[\begin{array}{ccc}
\cos \gamma & 0 & -\sin \gamma \\
0 & 1 & 0 \\
\sin \gamma & 0 & \cos \gamma
\end{array}\right] .
$$

La décomposition (7) peut être appliquée, en particulier, au vecteur déplacement mécanique $\hat{u}$ relatif à un problème quelconque traité dans $\hat{R}$. On désigne par $u$ le tenseur ainsi élaboré $[3,8,14]$.

Reprenons les équations de départ (1) et (2), en y effectuant les changements de variables simultanés $\hat{x}_{j} \rightarrow x_{i}$ et $\hat{u}_{r} \rightarrow u_{s}$. Nous obtenons de nouvelles équations

$$
\hat{C}_{j i k l} \frac{\partial^{2} u_{r}}{\partial x_{s} \partial x_{t}} Q_{r l} R_{t j} R_{s k}=\rho Q_{q i} \ddot{u}_{q} .
$$

Ces équations ne constituent pas exactement les équations d'équilibre dynamique dans le repère $\mathcal{R}$, car les $u_{i}$ ne sont pas les composantes du déplacement mécanique dans $\mathcal{R}$. A partir de (10) nous obtenons de nouvelles équations, mathématiquement équivalentes à (1) et (2), en effectuant le produit contracté de (10) par $Q_{p i}$ et en simplifiant ensuite, à l'aide de la relation $Q_{q 1} Q_{p}=\delta_{q p}$ traduisant l'orthogonalité de la matrice $Q$. Les nouvelles équations dites " équa- 
tions transformées ", peuvent être mises sous la forme condensée :

$$
T_{t p}=C_{t p r s} \frac{\partial u_{r}}{\partial x_{s}} ; \quad \frac{\partial T_{t p}}{\partial x_{t}}=\rho \ddot{u}_{p}
$$

Cette présentation s'accompagne de l'adoption des notations suivantes :

$$
T_{t p}=R_{t m} Q_{p n} \hat{T}_{m n} ; \quad C_{t p r s}=R_{t j} Q_{p i} Q_{r l} R_{s k} \hat{C}_{i j k l} .
$$

Il convient de prendre garde au fait que $T_{t p}$ n'est pas un élément du tenseur des efforts dans le repère $\mathcal{R}$, au sens usuel. Un tel tenseur $\mathcal{C}$ s'exprimerait différemment en fonction des $\hat{T}_{m m}$ :

$$
\mathcal{C}_{t p}=R_{t m} R_{p n} \hat{T}_{m n} .
$$

On peut émettre une remarque de même nature au sujet des coefficients transformés $C_{\text {tprs }}$. La transformation effectuée, dont la signification physique n'est pas évidente, présente néanmoins un intérêt majeur : les coefficients $C^{\alpha}$ régissant la propagation d'épaisseur dans une plaque infinie y apparaissent très aisément. En effet, en observant que $R_{2 k}=\delta_{2 k}$ et $R_{2 j}=\delta_{2 j}$, nous obtenons :

$$
C_{2 p r 2}=Q_{p i} Q_{r l} \hat{C}_{i 22 l}=Q_{p i} Q_{r l} \hat{C}_{2 i 2 l}
$$

par suite de la symétrie des coefficients habituels $\hat{C}_{i j k l}$. Rappelons (4) et (6). Il vient

$$
\hat{C}_{2 i 2 l} A_{l}^{r}=C_{2 i 2 l} Q_{r l}=C^{r} Q_{r i} .
$$

Grâce à l'orthogonalité de la matrice $Q$, on obtient :

$$
C_{2 p r 2}=Q_{p i} Q_{r i} C^{r}=\delta_{p r} C^{r}
$$

La symétrie des coefficients transformés $C_{i j k l}$ est faible. On remarque seulement que $C_{i j k l} \stackrel{=}{=} C_{l k j i i^{*}}$ Nous pouvons adopter la loi suivante pour comprimer les indices :

$$
\begin{array}{lll}
11 \rightarrow 1, & 22 \rightarrow 2, & 33 \rightarrow 3 \\
12 \rightarrow 6, & 23 \rightarrow 4, & 31 \rightarrow 5 \\
21 \rightarrow 9, & 32 \rightarrow 7, & 13 \rightarrow 8 .
\end{array}
$$

En tenant compte de la symétrie déjà signalée et de (16), nous obtenons les équations transformées suivantes, sous une forme développée et en adoptant la notation dite d'Einstein, pour les dérivations partielles par rapport aux $x_{i}$ :

$$
\begin{aligned}
& C^{(1)} u_{1,22}+2 C_{16} u_{1,12}+2 C_{56} u_{1,23}+C_{11} u_{1,11}+2 C_{18} u_{1,13}+C_{58} u_{1,33}+ \\
& +\left(C_{12}+C_{66}\right) u_{2,12}+\left(C_{28}+C_{76}\right) u_{2,23}+\left(C_{17}+C_{86}\right) u_{3,12}+\left(C_{36}+C_{48}\right) u_{3,23} \int_{C_{19}} u_{2,11}+ \\
& +\left(C_{14}+C_{59}\right) u_{2,13}+C_{54} u_{2,33}+C_{15} u_{3,11}+\left(C_{13}+C_{55}\right) u_{3,13}+C_{38} u_{3,33}=\rho \ddot{u}_{1} \\
& C^{(2)} u_{2,22}+2 C_{29} u_{2,12}+2 C_{24} u_{2,23}+C_{69} u_{2,11}+2 C_{64} u_{2,13}+C_{74} u_{2,33}+ \\
& +\left(C_{12}+C_{66}\right) u_{1,12}+\left(C_{28}+C_{76}\right) u_{1,23}+\left(C_{25}+C_{49}\right) u_{3,12}+\left(C_{23}+C_{44}\right) u_{3,23} \int+C_{19} u_{1,11}+ \\
& +\left(C_{14}+C_{59}\right) u_{1,13}+C_{54} u_{1,33}+C_{65} u_{3,11}+\left(C_{39}+C_{75}\right) u_{3,13}+C_{34} u_{3,33}=\rho \ddot{u}_{2} \\
& C^{(3)} u_{3,22}+2 C_{45} u_{3,12}+2 C_{37} u_{3,23}+C_{85} u_{3,11}+2 C_{35} u_{3,13}+C_{33} u_{3,33}+ \\
& +\left(C_{17}+C_{86}\right) u_{1,12}+\left(C_{36}+C_{48}\right) u_{1,23}+\left(C_{25}+C_{49}\right) u_{2,12}+\left(C_{23}+C_{44}\right) u_{2,23} \int_{15}+C_{15} u_{1,11}+ \\
& +\left(C_{13}+C_{55}\right) u_{1,13}+C_{38} u_{1,33}+C_{65} u_{2,11}+\left(C_{39}+C_{75}\right) u_{2,13}+C_{34} u_{2,33}=\rho \ddot{u}_{3} .
\end{aligned}
$$

A ce point, notre démarche s'éloigne de celle adoptée en [3].

Dans le cas d'une propagation purement en épaisseur, les équations (18-20) se réduisent très simplement à :

$$
\left.\begin{array}{l}
C^{(1)} u_{1,22}=\rho \ddot{u}_{1} \\
C^{(2)} u_{2,22}=\rho \ddot{u}_{2} \\
C^{(3)} u_{3,22}=\rho \ddot{u}_{3}
\end{array}\right\} .
$$

L'intérêt de la transformation (12) réside donc essentiellement dans le fait que les équations de propagation des ondes planes deepaisseur prennent alors une forme découplée particulièrement simple. Il devient ainsi plus facile de traiter un problème tendant « à la limite » vers une propagation d'épaisseur pure. En ce cas, au voisinage

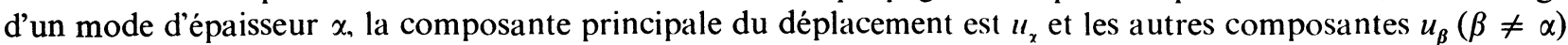
interviennent dans léquation de $u_{\alpha}$ essentiellement par lintermédiaire des termes tels que $u_{\beta .2 k}$. Ceci définit un état de quasi-vibration d'épaisseur pour les trois familles modales $(x=1,2,3)$, état que lon peut décrire avec une bonne approximation, en négligeant tous les termes qui figurent ì droite du signe $\int$ au premier membre 
des équations (18) à (20). Le quartz n'étant pas très piézoélectrique, les coefficients piézoélectriques interviennent faiblement sur le résultat du calcul des fréquences de résonance. Nous suivons donc la démarche habituelle de H. F. Tiersten en admettant que, en régime de quasi-vibration d'épaisseur, la distribution de potentiel électrostatique est proche de celle relevée dans une plaque infinie. Cela revient à modifier seulement les valeurs des $C^{\alpha}$, en tenant compte du durcissement imposé par la piézoélectricité [9], mais en négligeant les couplages entre modes qui résultent des conditions aux limites électriques. Ces couplages ont, sur la fréquence, un effet considérablement plus faible que les couplages mécaniques étudiés ici. C'est pourquoi nous ne tiendrons pas compte de la piézoélectricité dans l'établissement de la méthode de perturbation présentée dans ce qui suit.

Bien que les équations (11) ne soient pas tout à fait conventionnelles, il est possible de leur adjoindre des conditions aux limites. Pour un problème homogène aux limites, ces conditions sont :

$$
n_{t} T_{t p}=0 \text { sur un élément de la surface limite de normale } \mathbf{n} \text { dans } \mathcal{R} .
$$

L'orthogonalité des matrices $R$ et $Q$ et la linéarité de la transformation, garantissent l'équivalence de l'ensemble équations de propagation et conditions aux limites (11) et (22), avec l'ensemble correspondant exprimé conventionnellement dans $\mathcal{R}$. La validité des expressions établies jusqu'ici est indépendante de la valeur de la rotation $\gamma$.

Plaçons-nous dans le cas d'un résonateur réel. Si ses dimensions latérales sont assez grandes devant l'épaisseur, la polarisation de la vibration tend vers l'une ou l'autre des composantes $u_{i}$ et il y a tendance au découplage des modes. Les équations obtenues dans un cas limite seront donc :

$$
\left.\begin{array}{l}
C^{(1)} u_{1,22}+C_{11} u_{1,11}+C_{58} u_{1,33}+2 C_{16} u_{1,12}+2 C_{56} u_{1,23}+2 C_{18} u_{1,13}=\rho \ddot{u}_{1} \\
C^{(2)} u_{2,22}+C_{69} u_{2,11}+C_{74} u_{2,33}+2 C_{29} u_{2,12}+2 C_{24} u_{2,23}+2 C_{64} u_{2,13}=\rho \ddot{u}_{2} \\
C^{(3)} u_{3,22}+C_{85} u_{3,11}+C_{33} u_{3,33}+2 C_{45} u_{3,12}+2 C_{37} u_{3,23}+2 C_{35} u_{3,13}=\rho \ddot{u}_{3}
\end{array}\right\} .
$$

Nous ne savons pas résoudre les équations (23) avec les conditions aux limites associées. Dans le cas de résonateurs plans-convexes ou biconvexes, nous pouvons résoudre, en première approximation, les équations à variables séparables suivantes :

$$
\left.\begin{array}{l}
C^{(1)} u_{1,22}+C_{11} u_{1,11}+C_{58} u_{1,33}=\rho \ddot{u}_{1} \\
C^{(2)} u_{2,22}+C_{69} u_{2,11}+C_{74} u_{2,33}=\rho \ddot{u}_{2} \\
C^{(3)} u_{3,22}+C_{85} u_{3,11}+C_{33} u_{3,33}=\rho \ddot{u}_{3}
\end{array}\right\} .
$$

Pour que (24) constitue une bonne approximation de (23), on peut choisir $\gamma$ en respectant au mieux les symétries du problème. Lorsqu'on s'intéresse à un mode de la famille $\alpha(\alpha=1,2,3)$, il est logique de choisir $\gamma=-\operatorname{arctg}(Q \alpha 3 / Q \alpha 1)$ ou $\gamma=+\operatorname{arctg}(Q \alpha 1 / Q \alpha 3)$ de manière à faire coïncider l'un des axes du repère avec la projection de la polarisation $\mathbf{A}^{\alpha}$ dans le plan de la plaque. Un tel choix [5] n'optimise que l'accès à une seule famille modale, considérée comme principale (Cf. Fig. 2), mais de petites erreurs sur la description des modes

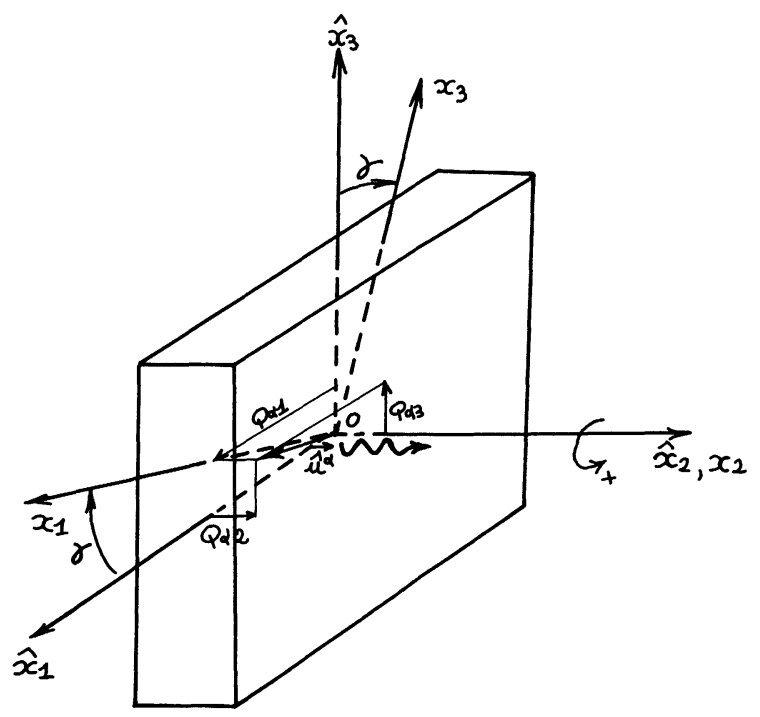

Fig. 2. - Choix de la rotation $\gamma$.

[Choice of the $\gamma$ rotation.] 
des autres familles se répercutent faiblement par couplage, sur les modes de la famille étudiée principalement. La symbolisation est arbitraire : nous adoptons $\alpha=1$ pour le cisaillement lent (mode $\mathrm{C}$ habituel [9]), $\alpha=2$ pour la compression (A) et $\alpha=3$ pour le cisaillement rapide (B).

Les équations simplifiées (24) doivent être vérifiées par les solutions du modèle utilisé en approximation initiale dans la méthode de perturbation. Ce modèle peut aujourd'hui être considéré comme classique [5].

Dans le cas d'une plaque d'épaisseur lentement variable, on tient compte des seules conditions aux limites sur les grandes faces. L'origine est prise au centre du résonateur, d'épaisseur $2 h$. Par suite du découplage des modes, la seule condition à la limite qui soit non triviale se résume à ((11) et (16)) :

$$
C^{\alpha} u_{\alpha, 2}=0 \text { pour } x_{2}=\mp h \Leftrightarrow T_{2 p}=0 \text { pour } x_{2}=\mp h .
$$

Si $u_{\alpha, 22}$ est très grand devant $u_{\alpha, 11}$ et $u_{\alpha, 33}$, les solutions sont du type suivant :

ou bien

$$
u_{\alpha}=A_{\alpha}\left(x_{1}, x_{3}\right) \sin \frac{n \pi x_{2}}{2 h} \mathrm{e}^{j \omega t}
$$

$$
u_{\alpha}=B_{\alpha}\left(x_{1}, x_{3}\right) \cos \frac{q \pi x_{2}}{2 h} \mathrm{e}^{j \omega t}
$$

où $n$ est un entier pair et $q$ un entier impair. Ces nombres définissent le rang de partiel de la vibration. Celle-ci est supposée de parité définie. Seuls les partiels impairs sont excitables piézoélectriquement de façon simple, mais les partiels pairs peuvent être excités mécaniquement, de façon directe ou par couplage. Par report de (26) dans l'équation correspondante de (24), on obtient une équation de dispersion, obéissant au schéma général :

$$
\left(\rho \omega^{2}-C^{\alpha} \frac{k^{2} \pi^{2}}{4 h^{2}}\right) u_{\alpha}+K u_{\alpha, 11}+L u_{\alpha, 33}=0 .
$$

Dans le cas d'un résonateur plan convexe, d'épaisseur maximale au centre $2 h_{0}$ et de rayon de courbure $R$, on obtient par développement limité de $h\left(x_{1}, x_{3}\right)$, une équation qui se sépare en deux équations d'Hermite :

avec :

$$
\left.\begin{array}{l}
K \frac{F^{\prime \prime}}{F}-C^{\alpha} \frac{k^{2} \pi^{2}}{4 R h_{0}^{2}} x_{1}^{2}=-\lambda \\
L \frac{G^{\prime \prime}}{G}-C^{\alpha} \frac{k^{2} \pi^{2}}{4 R h_{0}^{2}} x_{3}^{2}=-\mu
\end{array}\right\}
$$

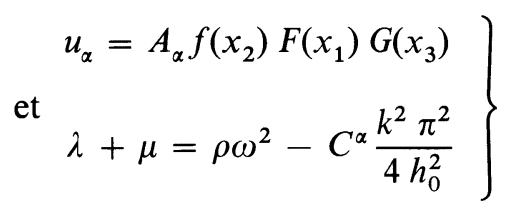

Les équations (28) admettent des solutions discrètes pour :

$$
\left.\begin{array}{l}
\lambda=(2 m+1) C^{\alpha} K \frac{k \pi}{4 h_{0}^{2}} \sqrt{\frac{2 h_{0}}{R}} \\
\mu=(2 p+1) C^{\alpha} L \frac{k \pi}{4 h_{0}^{2}} \sqrt{\frac{2 h_{0}}{R}}
\end{array}\right\}
$$

Si $\omega_{k}$ désigne la pulsation à la résonance de la plaque infinie correspondante au résonateur étudié et d'épaisseur $2 h_{0}$, fonctionnant sur le $k$-ième rang de partiel, on obtient finalement :

$$
\frac{\omega_{k m p}^{2}-\omega_{k}^{2}}{\omega_{k}^{2}}=\frac{1}{k \pi} \sqrt{\frac{2 h_{0}}{R}}\left\{(2 m+1) \sqrt{\frac{k}{C^{\alpha}}}+(2 p+1) \sqrt{\frac{L}{C^{\alpha}}}\right\} .
$$

Chaque fréquence de résonance et chaque figure modale sont associées à quatre nombres : un nombre qualifiant la famille modale (ici $\alpha$ ), le rang de partiel $k$, un nombre qualifiant le « rang d'anharmonicité » dans la direction $\mathbf{x}_{1}$ (ici $m$ ), un nombre analogue pour la direction $\mathbf{x}_{3}$ (ici $p$ ). Les fonctions d'Hermite tendent à s'annuler pour les valeurs élevées de $x_{1}$ et $x_{3}$, mais d'autant moins vite que $m$ et $p$ sont grands. Lorsque l'amplitude de la vibration s'annule assez vite, la solution vérifie, a posteriori, les conditions aux limites sur le pourtour de la plaque. Seuls les modes de ce type possèdent une existence physique certaine, ce qui est confirmé par l'expérience [10]. 


\section{Méthode de perturbation.}

Considérons un milieu élastique très étendu dans toutes les directions, siège d'une vibration de composantes $u_{i}$, résultant de l'action d'une répartition de sources élémentaires $f_{j}$. La vibration en un point $P$ pourra être mise sous la forme :

$$
u_{i}(P)=\int_{V} G_{j}^{i}(P, Q) f_{j}(Q) \mathrm{d} V(Q) .
$$

$V$ est le volume du matériau, assez étendú pour que les conditions aux limites n'influent pas sur ce qui se passe en un point $P$ situé au cour du matériau. Les points tels que $P$ sont appelés points-champs, les points tels que $Q$ sont appelés points-sources. $G_{j}^{i}$ définit le tenseur de Green, transposition tensorielle de la notion de fonction de Green rencontré en Electrostatique [11]. L'indice supérieur est relatif au champ, l'indice inférieur est relatif à la source. Dans un premier temps, nous allons trouver l'équation fondamentale vérifiée par le tenseur de Green; ensuite nous introduirons l'effet des conditions aux limites indispensables au traitement des problèmes de résonateurs. Enfin, nous donnerons l'expression générale du déplacement mécanique pour le problème étudié, d'où l'on tire les fréquences propres " perturbées " du résonateur.

Les équations (18), (19), (20) peuvent être écrites sous la forme :

$$
C_{t p r s}^{0} \frac{\partial^{2} u_{r}}{\partial x_{s} \partial x_{t}}+\rho \omega^{2} u_{p}=-C_{t p r s}^{+} \frac{\partial^{2} u_{r}}{\partial x_{s} \partial x_{t}} \text {. }
$$

Les coefficients $C_{t p r s}^{0}$ sont ceux qui apparaissent dans (24) et (25). Les coefficients $C_{t p r s}^{+}$sont les autres coefficients figurant dans (18), (19), (20). Il faut noter le fait que, dans (33) $u_{r}$ est une composante du déplacement total et non une composante du déplacement obtenu pour la solution non perturbée, ou simplifiée. Le fondement de la démarche consiste à considérer que l'effet des coefficients élastiques initialement négligés est localement assimilable à celui provoqué par une répartition de forces de volume.

On peut donc remplacer (33) par :

$$
C_{t p r s}^{0} \frac{\partial^{2} u_{r}}{\partial x_{s} \partial x_{t}}+\rho \omega^{2} u_{p}=f_{p}
$$

Cette équation doit être vraie en tout point $P$ du matériau. Admettons qu'il soit possible de trouver $G_{j}^{i}$ définie en (32). En exprimant $u_{r}$ et $u_{p}$ à l'aide de $G_{j}^{i}$, on obtient de nouvelles équations équivalentes à (34) :

$$
\begin{aligned}
& \int_{V}\left(C_{t p r s}^{0} G_{k}^{r, s t}+\rho \omega^{2} G_{k}^{p}\right) f_{k} \mathrm{~d} V(Q)=f_{p}(P) \\
& (p=1,2,3) . \quad(35) \\
& \text { Dans cette équation, } G_{k}^{r, s t} \text { signifie } \frac{\partial^{2} G_{k}^{r}(P, Q)}{\partial x_{s} \partial x_{t}} \text { à } Q
\end{aligned}
$$

constant. Le volume d'intégration pouvant être quelconque, les équations (35) peuvent être remplacées par

$$
C_{t p r s}^{0} G_{k}^{r, s t}+\rho \omega^{2} G_{k}^{p}=\delta_{k}^{p} \delta(P-Q)
$$

où nous adoptons pour le symbole de Kronecker $\delta_{k}^{p}$ une notation cohérente avec celle choisie pour le tenseur de Green. $\delta(P-Q)$ symbolise la distribution de Dirac. En postulant le principe de réciprocité selon lequel l'effet en $P$ d'une source placée en $Q$ est le même que celui en $Q$ d'une source placée en $P$ et en sachant que la distribution de Dirac est symétrique par rapport aux points champs et sources, on peut inverser la position haute ou basse des indices. On obtient alors l'équation fondamentale :

$$
C_{t p r s}^{0} G_{r, s t}^{k}+\rho \omega^{2} G_{p}^{k}=\delta_{p}^{k} \delta(P-Q) .
$$

A un signe conventionnel près, cette équation, complétée par l'introduction de la piézoélectricité, est donnée par B. K. Sinha [6] et H. F. Tiersten [7], mais comme un postulat, sans autre justification que la citation en référence de l'ouvrage [11], où le lecteur la cherche en vain sous cette forme précise. Pour sa part, l'auteur préfère partir de (32), qui paraît définir la fonction de Green plus physiquement que (37), qui doit être considéré comme un corollaire. Ceci correspond d'ailleurs très fidèlement à la démarche suivie dans l'ouvrage de Morse et Feshback ([11] chapitre 7). En suivant ces auteurs on admet que la fonction de Green permettant d'obtenir en un point du matériau le champ créé par des sources situées sur sa surface limite est de même nature que celle relative à des sources situées dans le volume du matériau. C'est pourquoi l'équation (37) peut être maintenue lorsqu'on désire prendre en compte l'effet des conditions aux limites. Il nous reste cependant à préciser l'équation intégrale reliant $u_{i}(P)$ à $G_{j}^{i}(P, Q)$ en présence de ces conditions.

Multiplions tensoriellement (37) par $u_{p}(Q)$ et intégrons sur le volume $V$. De même, nous pouvons effectuer le produit contracté de (33) $\operatorname{par} G_{p}^{k}(P, Q)$, en intégrant ensuite sur $V$.

En retranchant l'équation ainsi obtenue à la première et en utilisant la définition de la distribution de Dirac, on trouve :

$$
u_{k}(P)=\int_{V}\left(T_{t p, t}^{0 k} u_{p}-T_{t p, t}^{0} G_{p}^{k}-T_{t p, t}^{+} G_{p}^{k}\right) \mathrm{d} V(Q)
$$

avec l'adoption des notations suivantes :

$$
\left.\begin{array}{l}
T_{t p}=T_{t p}^{0}+T_{t p}^{+} \\
T_{t p}^{0}=C_{t p r s}^{0} u_{r, s} \\
T_{t p}^{0 k}=C_{t p r s}^{0} G_{r, s}^{k}
\end{array}\right\} .
$$

L'équation (38) est toujours vraie. Jusqu'ici, aucune hypothèse n'a été émise au sujet de l'existence de 
solutions orthogonales, pour le problème simplifié, et au sujet d'éventuelles symétries présentées par les coefficients élastiques. Pour appliquer le formalisme usuel de perturbation à un modèle utilisant les coefficients transformés, il convient de modifier la forme de (38). A cet effet, on observe que grâce à la symétrie de ces coefficients par rapport au renversement de l'ordre des indices, symétrie que nous avons déjà mentionnée, les quantités $T_{t p}^{0} G_{p, t}^{k}$ et $T_{t p}^{0 k} u_{p, t}$ sont égales. En ce cas précis, l'identité suivante se trouve vérifiée :

$$
T_{t p, t}^{0 k} u_{p}-T_{t p, t}^{0} G_{p}^{k}=\left(T_{t p}^{0 k} u_{p}-T_{t p}^{0} G_{p}^{k}\right)_{, t}
$$

En appliquant à (38) le théorème de la divergence, on obtient alors :

$$
\begin{aligned}
u_{k}(P)=\int_{S}\left(T_{t p}^{0 k} u_{p}-T_{t p}^{0} G_{p}^{k}\right) n_{t} \mathrm{~d} S(Q)- \\
-\int_{V} T_{t p, t}^{+} G_{p}^{k} \mathrm{~d} V(Q)
\end{aligned}
$$

où $n_{t}$ est une composante de la normale unitaire à la surface limite. On peut simplifier un peu (41) dans le cas où l'on traite un problème dans lequel les contraintes, et par suite les contraintes transformées, sont nulles sur la surface limite. On remarque que :

$$
\begin{aligned}
\int_{V} T_{t p, t}^{+} G_{p}^{k} \mathrm{~d} V(Q)= & \int_{S} T_{t p}^{+} G_{p}^{k} n_{t} \mathrm{~d} S- \\
& -\int_{V} T_{t p}^{+} G_{p, t}^{k} \mathrm{~d} V(Q)
\end{aligned}
$$

En rassemblant les termes en $T_{t p}^{+} n_{t}$ et $T_{t p}^{0} n_{t}$, puis en utilisant $(39-1)$ et la nullité de la contrainte totale $T_{t p}$ sur la surface, nous obtenons :

$u_{k}(P)=\int_{S} T_{t p}^{0 k} u_{p} n_{t} \mathrm{~d} S(Q)+\int_{V} T_{t p}^{+} G_{p, t}^{k} \mathrm{~d} V(Q)$.

Cette équation donne la forme du déplacement mécanique. Pour expliciter le tenseur de Green, nous devons recourir à l'orthogonalité des fonctions propres obtenues comme solutions du problème simplifié. Celle-ci résulte de celle des fonctions d'Hermite et des lignes trigonométriques intervenant dans ces solutions. Afin de distinguer ces solutions, nous les qualifierons par un indice supérieur symbolique $\alpha$ ou $\beta$, résumant les quatre nombres caractéristiques mentionnés à la fin du paragraphe 2. Dans la suite, nous sous-entendrons le fait que les fonctions propres $u_{i}^{\alpha}$ ou $u_{i}^{\beta}$ sont normées de manière à vérifier la relation d'orthogonalité sous la forme suivante :

$$
\int_{V} \rho u_{i}^{\alpha} u_{i}^{\beta} \mathrm{d} V=\delta_{\alpha \beta}
$$

Le calcul des fonctions de Green est alors classique [11]. On développe $G_{p}^{k}$ sur la base des $u_{p}^{\alpha}$. A cet effet, on pose :

$$
G_{p}^{k}(P, Q)=K_{\alpha}^{k}(P) u_{p}^{\alpha}(Q)
$$

sans omettre de sommer sur $\alpha$. On obtient alors, en se souvenant de $(39-3)$ :

$$
T_{t p, t}^{0}=C_{t p r s}^{0} K_{\alpha}^{k} u_{r, s t}^{\alpha}=-\rho \omega_{\alpha}^{2} K_{\alpha}^{k} u_{p}^{\alpha} .
$$

(On applique la définition même des solutions $u_{p}^{\alpha}$ du problème simplifié, correspondant aux fréquences propres $\omega_{\alpha}$ ). On reporte (46) dans l'équation de base (36) et, par multiplication par $u_{p}^{\beta}$ et par intégration sur $V$, on obtient facilement :

$$
\int_{V} \rho\left(\omega^{2}-\omega_{\alpha}^{2}\right) K_{\alpha}^{k}(P) u_{p}^{\alpha}(Q) u_{p}^{\beta}(Q) \mathrm{d} V(Q)=u_{k}^{\beta}(P)
$$

$K_{\alpha}^{k}$ ne dépendant pas de $Q$ peut être extrait. En rappelant (44), on obtient enfin

$$
K_{\beta}^{k}=\frac{u_{k}^{\beta}(P)}{\omega^{2}-\omega_{\beta}^{2}} .
$$

On exprime alors les fonctions de Green relatives au déplacement $G_{p}^{k}$ et aux contraintes $T_{t p}^{0 k}$ en fonction des déplacements propres simplifiés.

En particulier :

$$
T_{t p}^{0 k} n_{t}=K_{\beta}^{k} C_{t p r s}^{0} u_{r, s}^{\beta} n_{t} .
$$

Comme le problème simplifié, dont les $u_{r}^{\beta}$ sont des solutions, correspond aussi à des conditions aux limites homogènes, la quantité $C_{t p r s}^{0} u_{r, s}^{\beta} n_{t}$ est nulle. Nous obtenons donc la forme rigoureuse ultime pour le déplacement mécanique :

$$
u_{k}(P)=K_{\beta}^{k} \int_{V} T_{t p}^{+} u_{p, t}^{\beta} \mathrm{d} V
$$

Dans cette équation, on n'omettra pas la sommation sur $\beta$. Cette équation, rigoureuse, nous indique que la solution exacte ne dépend que des solutions du problème simplifié et des coefficients élastiques négligés au départ. On pose :

$$
\begin{aligned}
& T_{t p}^{+\alpha}=C_{t p r s}^{+} u_{r, s}^{\alpha} \\
& Q_{\alpha \beta}=\int_{V} T_{t p}^{+\alpha} u_{p, t}^{\beta} \mathrm{d} V .
\end{aligned}
$$

Si la solution exacte $u_{k}$ n'est pas très éloignée d'une des solutions simplifiées, que nous désignerons par $u_{k}^{\alpha}$, on peut résoudre l'équation intégrale (50) $\left(u_{k}\right.$ apparaissant au $2^{\mathrm{e}}$ membre dans l'expression développée de $T_{t p}^{+}$) par un processus itératif de report de l'expression approchée des $u_{k}$ d'un membre de (50) dans l'autre. En partant de l'approximation d'ordre 
zéro, $u_{k} \sim u_{k}^{\alpha}$ et $\omega \sim \omega_{\alpha}$, et en distinguant le cas $\beta=\alpha$, on obtient au deuxième ordre :

$$
\begin{gathered}
u_{k}(P)=u_{k}^{\alpha}(P)+\sum_{\delta, \beta \neq \alpha} \frac{u_{k}^{\beta}}{\omega^{2}-\omega_{\beta}^{2}} \times \\
\times\left(Q_{\alpha \beta}+\frac{Q_{\alpha \delta} Q_{\delta \beta}}{\omega^{2}-\omega_{\delta}^{2}}\right)+\cdots \\
\omega^{2}-\omega_{\alpha}^{2}=Q_{\alpha \alpha}+\frac{Q_{\beta \alpha} Q_{\alpha \beta}}{\omega^{2}-\omega_{\beta}^{2}}+\cdots
\end{gathered}
$$

Dans un développement à l'ordre $(n)$, il convient de reporter dans l'expression de $u_{k}$ la valeur de $\omega$ obtenue à l'ordre $(n-1)$. Donc dans (52) il faut écrire $\omega^{2}=\omega_{\alpha}^{2}+Q_{\alpha \alpha}$. L'intérêt de la démarche suivie réside surtout dans la mise en évidence du rôle des conditions aux limites adjointes aux équations de propagation. Les applications présentées ici se limitent au calcul de perturbations sur la fréquence(53).

\section{Expression des $Q_{\alpha \beta}$.}

On étudie un mode désigné par le symbole $\alpha$. Il appartient à une famille modale, dont l'identificateur prend la valeur $i(i=1,2$ ou 3$)$. Le modèle simplifié nous donne assez exactement la solution des équations (24) dans le repère $\mathcal{R}$ associé à la $i$-ième famille modale, avec les conditions aux limites correspondantes. Par rapport à (24), nous avons introduit les termes supplémentaires suivants :

- Les termes en $u_{i, 12} u_{i, 23}$ et $u_{i, 13}$ dans la $i$-ième équation. Ils traduisent des couplages avec les autres modes de la famille à laquelle appartient $u_{i}^{\alpha}$.

- Les termes en $u_{k, 12} u_{k, 23}$ pour les deux valeurs de $k$ différentes de $i$, dans la $i$-ième équation. Ils correspondent aux termes en $u_{i, 12}$ et $u_{i, 23}$ dans les $k$-ièmes équations (Cf. (18)-(20)). Ils traduisent les couplages entre familles différentes de modes de quasi vibration d'épaisseur. Nous négligeons les termes tels que $u_{k, 13}$ ou $u_{k, 11}$ etc... dans la $i$-ième équation, à cause du rôle prédominant attribué à $x_{2}$.

Ce travail a été fait successivement pour les trois familles modales. On observe que pour les termes supplémentaires pris en considération, toutes les quantités $Q_{\alpha \alpha}$ sont nulles. En effet :

$$
Q_{\alpha \alpha}=\int_{V} u_{k, l}^{\alpha} C_{i j k l}^{+} u_{j, i}^{\alpha} \mathrm{d} V
$$

où l'on ne doit pas sommer sur $\alpha$. Dans le modèle de départ, seule la composante $u_{i}$ du mode $\alpha$ n'est pas nulle. Par conséquent, pour que $Q_{\alpha \alpha}$ ne soit pas nul, il doit prendre la forme :

$$
Q_{\alpha \alpha}=\int_{V} u_{i, l}^{\alpha} C_{j i i l}^{+} u_{i, j}^{\alpha} \mathrm{d} V
$$

où la sommation se fait seulement sur $j$ et $l$. Les solutions $\alpha$ étant de parité définie suivant les trois axes. la seule possibilité de non-nullité pour $Q_{\alpha x}$ est :

$$
\left.Q_{\alpha \alpha}=\int_{V}\left(u_{i, l}^{\alpha}\right)^{2} C_{l i i l}^{+} \mathrm{d} V \quad \text { (sommation sur } l\right) .
$$

Aucun des termes supplémentaires ne correspond à cette forme pour les coefficients élastiques.

Il faut donc poursuivre les développements au second ordre, en affectant au second membre des équations (52) et (53), la valeur $\omega_{\alpha}$ à $\omega$. Une propriété remarquable est la symétrie des éléments $Q_{\alpha \beta}$. Examinons par exemple la méthode de calcul de $Q_{\alpha \beta}$ et $Q_{\beta \alpha}$ pour des termes de couplage entre familles différentes. Si $\alpha$ appartient à la $i$-ième famille, les termes en question apparaissent sous la forme suivante, où l'on abandonne la convention de sommation des indices répétés :

$$
\begin{array}{ll}
\left(C_{l i k 2}^{+}+C_{2 i k l}^{+}\right) u_{k, 2 l} & \text { dans la } i \text {-ième équation } \\
\left(C_{2 k i l}^{+}+C_{l k i 2}^{+}\right) u_{i, 2 l} & \text { dans la } k \text {-ième équation } .
\end{array}
$$

Il faut donc tenir compte des contraintes supplémentaires $T_{l i}^{+}, T_{2 i}^{+}, T_{2 k}^{+}, T_{l k}^{+}$. Comme $k$ est nécessairement différent de $i$ et du fait que $u_{k}^{\alpha}$ est donc nul, on obtient, d'après (51) :

$$
Q_{\alpha \beta}=\int_{V}\left(T_{2 k}^{+\alpha} u_{k, 2}^{\beta}+T_{l k}^{+\alpha} u_{k, l}^{\beta}\right) \mathrm{d} V
$$

Pour les mêmes raisons :

$$
Q_{\beta \alpha}=\int_{V}\left(T_{l i}^{+\beta} u_{i, l}^{\alpha}+T_{2 i}^{+\beta} u_{i, 2}^{\alpha}\right) \mathrm{d} V
$$

Par suite de la symétrie des coefficients transformés par rapport au renversement de l'ordre des indices non réduits, on obtient :

$$
Q_{\alpha \beta}=Q_{\beta \alpha}=\int_{V}\left(C_{l i k 2}^{+} u_{i, l}^{\alpha} u_{k, 2}^{\beta}+C_{2 i k l}^{+} u_{i, 2}^{\alpha} u_{k, l}^{\beta}\right) \mathrm{d} V
$$

On vérifie tout aussi aisément la symétrie des éléments $Q_{\beta \alpha}$ dans le cas des couplages entre modes d'une même famille. Pour les termes en $u_{i, 2 k}$ on obtient :

$$
Q_{\alpha \beta}=Q_{\beta \alpha}=\int_{V} C_{k i i 2}^{+}\left(u_{i, 2}^{\alpha} u_{i, k}^{\beta}+u_{i, k}^{\alpha} u_{i, 2}^{\beta}\right) \mathrm{d} V
$$

Pour les termes en $u_{i, 13}$, on obtient :

$$
Q_{\alpha \beta}=Q_{\beta \alpha}=\int_{V} C_{1 i i 3}^{+}\left(u_{i, 3}^{\alpha} u_{i, 1}^{\beta}+u_{i, 1}^{\alpha} u_{i, 3}^{\beta}\right) \mathrm{d} V .
$$

En pratique, on peut se restreindre à l'étude de modes $\alpha$ impairs en $x_{2}$ et pairs en $x_{1}$ et $x_{3}$, ce qui correspond à une excitation par des électrodes monoblocs centrées sur les grandes faces de la plaque. 
On peut alors éliminer les termes $Q_{\alpha \beta}$ nuls par raison de symétrie. Par exemple, on constate que dans (59), on peut se limiter aux modes $u_{k}^{\beta}$ pairs en épaisseur, impairs en $x_{l}(l \neq 2)$ et pairs par rapport à la troisième variable dimensionnelle. Pour les termes tels que (60), on peut opérer une simplification de même nature. Enfin, dans le cas des termes en $u_{i, 13}$ l'intégration sur $x_{2}$ donne un résultat nul lorsque le rang de partiel du mode $u_{i}^{\beta}$ est différent de celui du mode $u_{i}^{\alpha}$, ce qui réduit la série (53) à une sommation double sur les rangs d'anharmonicité.

\section{Convergence des séries, critères de troncature.}

La précision des développements (52) et (53) en tant qu'approximation de l'équation intégrale (50) est déterminée par la rapidité de convergence de la méthode par rapport à l'ordre d'itération. L'étude de la convergence est difficile; de plus, le calcul numérique des développements correspondant à l'approximation du $3^{\mathrm{e}}$ ordre est très lourd. On peut seulement faire observer que la précision de l'approximation (du second ordre dans notre cas) dépend a priori, de la valeur des coefficients supplémentaires $C_{t p r s}^{+}$Comme la base modale des solutions mathé- matiques des équations simplifiées est infinie, nous sommes techniquement obligés de tronquer les développements (53), en limitant le nombre de termes $Q_{\alpha \beta}$ retenus dans le calcul numérique. Pour chaque coefficient ou groupe de coefficients $C_{t \text { prs }}^{+}$, la convergence des séries par rapport au rang de partiel des modes $\beta$ est assurée par la décroissance rapide des quantités $1 /\left(\omega_{\alpha}^{2}-\omega_{\beta}^{2}\right)$ et par celle des intégrales fonctions de l'épaisseur dans $\left(Q_{\alpha \beta}\right)$ : ces intégrales sont proportionnelles à : $\left[\left(\frac{1}{n-q}\right)+\left(\frac{1}{n+q}\right)\right]^{2}, n$ désignant le rang de partiel du mode $\alpha$ et $q$ celui du mode $\beta$. En pratique, nous avons limité le calcul aux modes $\beta$ tels que $\omega_{\beta} \leqslant 4 \omega_{\alpha}$.

La convergence par rapport aux rangs $m$ et $p$ définis en (31) est plus difficile à montrer. Les oscillations de plus en plus rapprochées des fonctions d'Hermite, à mesure que leur rang seélève, tendent à faire décroître les valeurs des intégrales calculées sur $x_{1}$ et $x_{3}$ dans $Q_{\alpha \beta}$.

En limitant la valeur maximale de la quantité $m+p$ relative aux modes $\beta$ retenus dans le calcul, nous avons obtenu les résultats du tableau I. Ils concernent les modes $\mathrm{C}$ d'une coupe SC $\left(\Phi=22^{\circ} 25^{\prime}\right.$, $\left.0=34^{\circ} 25^{\prime}\right)$ d'épaisseur $1.089 \mathrm{~mm}$. avec une face convexe de rayon $300 \mathrm{~mm}$.

\section{Tableau I. - Convergence des développements.}

[Convergence of expansions.]

\begin{tabular}{|c|c|c|c|c|c|}
\hline Mode $\alpha(n, m, p)$ & $3,0,0$ & $3,0,2$ & $3,2,0$ & $5,0,0$ & \\
\hline Fréquence initiale (Hz) & 5015376 & 5103110 & 5114490 & 8327980 & \\
\hline $\begin{array}{c}\text { Perturbation sur la fréquence } \\
\text { (Hz) }\end{array}$ & -4896 & -7723 & -22793 & +609 & $(m+p)_{\beta} \leqslant 10$ \\
& -4892 & -7702 & -22651 & +613 & $(m+p)_{\beta} \leqslant 7$ \\
& -4877 & -7799 & -22182 & +625 & $(m+p)_{\beta} \leqslant 5$ \\
& -4823 & -8473 & -20962 & +677 & $(m+p)_{\beta} \leqslant 3$ \\
\hline
\end{tabular}

Pour les séries de termes tels que (60) et (61), la précision relative d'une troncature ne dépend pas de la valeur des coefficients $C_{t p r s}^{+}$mais seulement des caractéristiques des solutions du problème simplifié. Celles-ci sont entièrement fixées par les coefficients $C_{t p r s}^{0}$ et les caractéristiques géométriques du résonateur. Dans le cas des termes tels que (59), la précision relative de la troncature dépend un peu de la valeur des coefficients supplémentaires $C_{l i k 2}^{+}$et $C_{2 i k l}^{+}$(sauf s'ils sont égaux ou si l'un des deux est nul).

Le tableau I peut donc être considéré comme assez typique. En pratique et compte tenu du temps de calcul numérique et de la nature des modes $\alpha$ étudiés, il est rarement utile de prendre en considération les modes $\beta$ caractérisés par une somme $m+p$ supérieure à cinq.

Il est possible d'adjoindre aux critères purement numériques, des critères de troncature d'origine physique. Connaissant le diamètre du résonateur, on constate que si une solution est insuffisamment piégée, elle ne vérifie pas les conditions aux limites sur le pourtour de la plaque avec une approximation suffisante [10]. De plus, pour les modes anharmoniques de rang très élevé, il peut survenir que les variations d'amplitude dans les directions $x_{1}$ et $x_{3}$ deviennent assez rapides pour que de tels modes théoriques soient en contradiction avec l'hypothèse initiale de quasi-vibration d'épaisseur. On peut ainsi limiter le nombre de modes $\beta$ retenus, mais dans ce cas, l'ensemble de ces modes ne constitue plus une base mathématiquement rigoureuse. Cette remarque est atténuée par le fait que la résolution de (50) par itération est intrinsèquement approximative. Nous recourons à ces troncatures supplémentaires, dont l'exposé détaillé sort du cadre de cet article, car elles permettent une réduction du temps de calcul, tout en 
modifiant peu la valeur des perturbations qu'on obtient.

\section{Résultats numériques.}

Le tableau II présente des résultats relatifs à un résonateur en coupe AT (coupe en simple rotation autour de l'axe cristallin $\mathbf{X}$ ). Ses caractéristiques sont
$\Phi=0^{\circ}, \theta=35^{\circ} 25^{\prime}, 2 h_{0} \sim 1,679 \mathrm{~mm}, R=150 \mathrm{~mm}$, diamètre de la pastille $15 \mathrm{~mm}$. Les modes résonnants sont ici les modes $\mathrm{C}$. Les perturbations de fréquence sont faibles et contribuent à améliorer l'accord global entre le spectre théorique et l'expérimental. L'analyse des vibrations par topographie aux rayons $\mathrm{X}$ a permis de constater que l'identification des modes est conforme au modèle de Wilson [12].

Tableau II. - Résultats pour une coupe AT.

[Results for AT cut.]

\begin{tabular}{|c|c|c|c|}
\hline Mode $\alpha(n, m, p)$ & f. Wilson $(\mathrm{Hz})$ & Perturbation $(\mathrm{Hz})$ & f. exp. (kHz) $[12]$ \\
\hline $5,0,0$ & 5000908 & +151 & 5000,0 \\
$5,2,0$ & 5113164 & -327 & 5114,4 \\
$5,0,2$ & 5100998 & -230 & 5100,2 \\
$7,0,0$ & 6979778 & -840 & 6977,0 \\
$7,2,0$ & 7092730 & -4626 & 7087 \\
$7,0,2$ & 7080455 & -765 & 7081 \\
$11,0,0$ & 10937427 & -906 & 10934 \\
$11,2,0$ & 11051023 & -4449 & 11044 \\
$11,0,2$ & 11038647 & -892 & 11037 \\
\hline
\end{tabular}

Les résultats du tableau III concernent les modes $\mathrm{C}$ d'une coupe $\mathrm{SC}\left(\Phi=22^{\circ} 26^{\prime}, \theta=34^{\circ} 25^{\prime}, 2 h_{0} \sim\right.$ $1,089 \mathrm{~mm}, R=300 \mathrm{~mm}$ ). Le tableau IV concerne les modes $B$ du même résonateur. En règle générale, les correctifs calculés sont beaucoup plus importants que pour une coupe AT de géométrie semblable, en raison de l'ordre de grandeur des coefficients $C_{t p r s}^{+}$. Pour le mode $\mathrm{C}$, les contributions prépondérantes à la perturbation proviennent des couplages $u_{1,12}, u_{2,12}$ et $u_{3,13}$. Ces contributions sont négatives, sauf celle fournie par $u_{3,23}$. Dans le cas du mode B, les contributions prépondérantes, toutes négatives, proviennent des couplages $u_{3,23}, u_{1,23}$ et $u_{2,23}$. Ceci est à l'origine des renversements obtenus dans l'ordre du spectre, ce qui rend l'identification des modes malaisée, sauf dans le cas des modes $(n, 0,0)$, isolés et présentant habituellement les meilleurs coefficients de qualité. Ces modes, utilisés dans les applications métrologiques, sont les moins perturbés par les couplages étudiês.

\section{Conclusion.}

Le modèle de base permet une description analytique des modes piégés qui offre l'avantage de la simplicité. Son extension aux coupes en double rotation [5] s'accompagne nécessairement d'une perte de précision. Quoique étant assez lourde dans sa mise en oeuvre, la méthode que nous proposons fournit un schéma de travail facilitant la détermination des limites du modèle de base. La démarche peut d'ailleurs s'appliquer à d'autres modèles simplifiés, analytiques.

Tableau III. - Résultats pour une coupe SC (mode C).

[Results for SC cut (C mode).]

\begin{tabular}{|c|c|c|c|}
\hline$(n, m, p)$ & f. Wilson $(\mathrm{Hz})$ & f. corrigée $(\mathrm{Hz})$ & f. exp. $(\mathrm{kHz})$ \\
\hline $3,0,0$ & 5015376 & 5010225 & 5000,00 \\
$3,2,0$ & 5111490 & 5079366 & 5082,23 \\
$3,0,2$ & 5103110 & 5085168 & 5088,95 \\
$5,0,0$ & 8327980 & 8328532 & $/$ \\
$5,2,0$ & 8424813 & 8418528 & 8398,98 \\
$5,0,2$ & 8416342 & 8423344 & 8405,56 \\
$5,4,0$ & 8520546 & 8505879 & 8483,57 \\
$5,2,2$ & 8512170 & 8511168 & 8490,32 \\
$5,0,4$ & 8503785 & 8510822 & 8499,20 \\
\hline
\end{tabular}


Tableau IV. - Résultats pour une coupe SC (mode B).

[Results for SC cut (B mode).]

\begin{tabular}{|c|c|c|c|}
\hline$(n, m, p)$ & f. Wilson $(\mathrm{Hz})$ & f. corrigée $(\mathrm{Hz})$ & f. exp. $(\mathrm{kHz})$ \\
\hline $3,0,0$ & 5507016 & 5497655 & 5469,69 \\
$3,2,0$ & 5592660 & 5581339 & 5535,53 \\
$3,0,2$ & 5613899 & 5565701 & 5561,40 \\
$5,0,0$ & 9145931 & 9140422 & 9094,85 \\
$5,2,0$ & 9232141 & 9224806 & 9161,81 \\
$5,0,2$ & 9253601 & 9226046 & 9183,42 \\
$5,4,0$ & 9317553 & 9308006 & 9216,00 \\
$5,2,2$ & 9338817 & 9311167 & 9262,09 \\
$5,0,4$ & 9360031 & 9311502 & 9269,71 \\
\hline
\end{tabular}

Le mécanisme de couplage introduit par chaque groupe de coefficients élastiques est nettement mis en évidence, même si la précision globale des correctifs calculés est susceptible de progrès. Une des causes d'imprécision peut résider dans la prise en considération des divers groupes de coefficients supplémentaires de façon à la fois simultanée et indépendante. Ce travail appelle des développements incluant notamment l'action de la température [13], et la prise en compte des termes figurant à droite du signe $\int$ dans les équations (18) à (20).

\section{Remerciements.}

L'auteur remercie F. Fichet qui lui a facilité l'élaboration des programmes de calcul numérique et fourni les résultats expérimentaux relatifs à la coupe $\mathrm{SC}$.

\section{Bibliographie}

[1] Fukuyo, H., Yokoyama, A., Ooura, N., Nonaka, S., Vibrations of biconvex circular AT cut Plate. Bulletin of Tokyo Institute of Technology 72/1965, mars 1966.

[2] Wilson, C. J., Vibration Modes of AT-cut Convex Quartz Resonators. J. Phys. D. : Appl. Phys. 7 (1974) 2449.

[3] Tiersten, H. F., Stevens, D. S., An analysis of Contoured SC-cut quartz Crystal resonators. Proceedings of Annual Frequency Control Symposium 1982 , p. 37.

[4] Tiersten, H. F., Linear Piezoelectric Plate vibrations (Plenum Press N.Y.) 1969.

[5] Bourquin, R., Nassour, D., Hauden, D., Amplitude Frequency Effect of SC cut Quartz Trapped Energy Resonators. Proceeding of Annual Frequency Control Symposium 1982, p. 200.

[6] Tiersten, H. F., Sinha, B. K., A perturbation Analysis of the Attenuation and dispersion of Surface waves. J. Appl. Phys. 49 (1978) 87.

[7] Tiersten, H. F., Perturbation Theory for Linear Electroelastic Equations for Small Fields Superposed on a Bias. J. Acoust. Soc. Am. 64 (3) (1978).
[8] Stevens, D. S., Tiersten, H. F., An analysis of SC cut Quartz Trapped Energy Resonators with Rectangular Electrodes. Proceedings of Annual Frequency Control Symposium 1981, p. 205.

[9] Dieulesaint, E., Royer, D., Ondes élastiques dans les solides. (Editeur Masson et Cie) 1974.

[10] ThIRARD, A., Etudes topographiques des vibrations de cisaillement d'épaisseur de Résonateurs à quartz. (Thèse D.I.-E.N.S.M.M.) Besançon 1980.

[11] Morse, P. M., Feshbach, H., Methods of Theoretical Physics. (Mc Graw Hill, N.Y.) 1953.

[12] Genestier, G., Application de la topographie par rayons $X$ à l'étude des modes de vibration dans un résonateur à ondes de volume. (Thèse D.I.-E.N.S.M.M.) Besançon 1982.

[13] Dulmet, B., Bourquin, R., Méthodes de détermination des courbes fréquence-température d'un résonateur à quartz vibrant en mode d'épaisseur. Revue Phys. Appl. 18 (1983) 619-624.

[14] KaUL, R. K., Mindlin, R. D., Vibrations of an Infinite, Monoclinic Crystal Plate at High Frequencies and Long Wavelengths. J. Acoust. Soc. Am. 34 (1962) 1895. 